\title{
Axonal TAU sorting requires the C-terminus of TAU but is independent of ANKG and TRIM46 enrichment at the AIS
}

\author{
Bell, M. ${ }^{1,2}$, Bachmann, S. ${ }^{1,2}$, Klimek, J. ${ }^{1,2}$, Langerscheidt, F. ${ }^{1,2,3}$, Zempel, H. ${ }^{1,2}$ \\ 1 Institute of Human Genetics, University Hospital Cologne, Kerpener Str. 34, 50931 Cologne, \\ Germany. \\ ${ }^{2}$ Center for Molecular Medicine Cologne (CMMC), Robert-Koch-Str. 21, 50931 Cologne, Germany. \\ ${ }^{3}$ FB03 Chemistry and Biotechnology, Campus Jülich, Fachhochschule Aachen, Heinrich-Mußmann- \\ Straße 1, 52428 Jülich, Germany.
}

Correspondence:

Michael Bell, Center of Molecular Medicine Cologne (CMMC), Robert-Koch-Str. 21, 50931 Cologne, michael.bell@uk-koeln.de.

Hans Zempel, Institute of Human Genetics, University Hospital Cologne, Kerpener Str. 34, 50931

Cologne, Germany, hans.zempel@uk-koeln.de

\section{Abstract}

Somatodendritic missorting of the axonal protein TAU is a hallmark of Alzheimer's disease and related tauopathies. Cultured rodent primary neurons and iPSC-derived neurons are used for studying mechanisms of neuronal polarity, including TAU trafficking. However, these models are expensive, time-consuming and/or require the sacrification of animals. In this study, we evaluated four differentiation procedures to generate mature neuron cultures from human SH-SY5Y neuroblastoma cells, in comparison to mouse primary neurons, and tested their TAU sorting capacity. We show that SH-SY5Y-derived neurons, differentiated with sequential RA/BDNF treatment, are suitable for investigating axonal TAU sorting. These human neurons show pronounced neuronal polarity, axodendritic outgrowth, expression of the neuronal maturation markers TAU and MAP2, and, importantly, efficient axonal sorting of endogenous and transfected human wild type TAU, similar to primary neurons. We demonstrate that axonal TAU enrichment requires the presence of the C-terminal half, as a C-terminus-lacking construct ( $\mathrm{N}$-term-TAU $\left.{ }^{\mathrm{HA}}\right)$ is not axonally enriched in both neuronal cell models. Moreover, SH-SY5Y-derived neurons do not show formation of a classical axon initial segment (AIS), indicated by the lack of Ankyrin G (ANKG) and tripartite motif-containing protein 46 (TRIM46) at the proximal axon, which suggests that successful axonal TAU sorting is independent of classical AIS formation. Taken together, our results suggest i) that SH-SY5Y-derived neurons are a valuable human neuronal cell model for studying TAU sorting, which is readily accessible at low cost and without animal need, and that ii) the mechanisms of axonal TAU targeting require the TAU C-terminal half but are independent of ANKG or TRIM46 enrichment at the proximal axon.

Key words: TAU sorting, Alzheimer's disease, SH-SY5Y-derived neurons, Neuronal polarity, ANKG, TRIM46 


\section{Introduction}

The microtubule-associated protein TAU is highly abundant in the axons of healthy human brain neurons ${ }^{1,2}$, where six isoforms are predominantly expressed ${ }^{3,4}$. By binding microtubule (MT) filaments, TAU regulates their assembly and disassembly ${ }^{5-}$ 7, and is involved in axonal outgrowth, plasticity, MT-dependent cargo transport and other cellular functions $\mathrm{s}^{8-12}$. The $\mathrm{C}$-terminal repeat domains and their flanking regions bind the MTs $\mathrm{s}^{13,14}$, while the $\mathrm{N}$-terminus acts as a spacer between adjacent MT filaments and mediates membrane interactions ${ }^{15-17}$. Missorting of highly phosphorylated TAU into the somatodendritic compartment and the formation of insoluble TAU aggregates ('neurofibrillary tangles') are major hallmarks of Alzheimer's disease (AD) and related tauopathies ${ }^{18-23}$. Subcellular TAU mislocalization leads to a lack of axonal TAU-MT interaction, depletion of stable MT filaments and, eventually, to disruption of axonal transport ${ }^{24-26}$. In the dendrites, abnormal amounts of hyperphosphorylated TAU trigger TTLL6-mediated MT destabilization and spine loss, FYN-mediated excitotoxicity, and other processes leading to postsynaptic degradation ${ }^{27-32}$.

Understanding the mechanisms of axonal TAU sorting in healthy neurons is a critical step towards the elucidation of pathological processes that accompany TAU missorting. Several mechanisms are thought to drive axonal TAU sorting ${ }^{33,34}$, including active anterograde TAU trafficking, selective axonal TAU-MT interactions, or active retrograde retention of axonal TAU. In this context, the axon initial segment (AIS), a key player for developing and maintaining neuronal polarity with Ankyrin G (ANKG) as a master organizer of the molecular structure ${ }^{35-37}$, may be relevant, as polarized MT filaments at the AIS are involved in motor protein-mediated soma-to-axontrafficking ${ }^{35,38}$, presumably including TAU transit ${ }^{33,34}$. The tripartite motif containingprotein 46 (TRIM46) may also be involved into anterograde TAU sorting by regulating the MT architecture at the AIS ${ }^{39}$. Moreover, recent studies found direct evidence for a retrograde TAU diffusion barrier within the AIS that is compromised by MT disassembly ${ }^{40,41}$. Notably, little is yet known about the intrinsic components of TAU, i.e. functional domains, interaction motifs, posttranslational modifications and relevant TAU interactions, although some recent studies in rodent primary neurons assumed major roles for phosphorylation sites within the Proline-rich region and for the very $\mathrm{N}$ terminal domain of TAU, but only a minor role for the four MT-binding repeat domains $^{42,43}$.

Which neuronal cell models are available to address these key aspects of TAU sorting and missorting? Two commonly used cellular systems are rodent primary neuron cultures ${ }^{40-43}$ and recently also human induced pluripotent stem cell (iPSC)-derived neurons ${ }^{44-48}$. Both systems have considerable limitations. Rodent primary neuronal cultures require the use and sacrification of animals, and rodent TAU differs in several aspects from human TAU, e.g. regarding the isoform expression pattern ${ }^{49}$. In addition, the human cellular environment, i.e. the entire sorting machinery and interaction partners may differ from that in humans. Overexpressed human TAU often appears uniformly distributed in rodent primary neurons ${ }^{50}$. IPSC-derived neurons overcome many of these issues, but their generation is expensive, time-consuming and results 
in mixed cultures with variable homogeneity and differentiation efficiency ${ }^{51-53}$. The neuroblastoma cell line SH-SY5Y is human-derived ${ }^{54}$ and cells can be differentiated with various substances into polarized neuronal cells (SH-SY5Y-derived neurons) at low cost ${ }^{55}$. SH-SY5Y-derived neurons express common neuronal maturation marker proteins $^{56-61}$, including all six major human TAU isoforms ${ }^{62,63}$, show proper axonal TAU localization, and mimic the phosphorylation state of TAU in the adult human brain ${ }^{64-67}$. In brief, SH-SY5Y-derived neurons unite the benefits of a cell line - i.e. robust maintenance, fast growth rates, the saving of animal resources and simple accessibility for stable genetic engineering ${ }^{68,69}$ - with the higher scientific relevance of primary and iPSC derived neurons. In this study, we evaluated four differentiation procedures of SH-SY5Y cells for generating neuronal cells and tested their suitability for the investigation of TAU sorting. Mouse primary forebrain neurons were used as a reference neuronal cell model. We show that RA/BDNF-treated SH-SY5Y-derived neurons achieve pronounced cellular polarity, axonal outgrowth, strong upregulation of the neuronal markers TAU and MT-associated protein 2 (MAP2), and, importantly, efficient axonal sorting of endogenous TAU and overexpressed human TAU (ON3RTAU $\left.{ }^{H A}\right)$. In contrast, TAU without the C-terminal half $\left(\mathrm{N}\right.$-term-TAU $\left.{ }^{\mathrm{HA}}\right)$ is not axonally enriched in SH-SY5Y-derived and mouse primary neurons. Of note, SH-SY5Y-derived neurons do not show enrichment of ANKG and TRIM46 at the proximal axon despite successful axonal TAU targeting, suggesting an ANKG- and TRIM46-independent mechanism of axonal TAU enrichment. In brief, our results give evidence that i) $\mathrm{SH}$ SY5Y-derived neurons show successful axonal sorting of endogenous and transfected human TAU, ii) the $\mathrm{N}$-terminus of TAU is not sufficient for proper axonal sorting, and ii) the axonal targeting of endogenous and 0N3R-TAU ${ }^{H A}$ is independent of ANKG, a master organizer of the AIS molecular structure, and TRIM46, a AIS-located MTorganizing protein. 


\section{Methods}

\section{Molecular biology}

For experiments with endogenous and transfected TAU, an expression vector, which harbored the cDNA encoding for the tdTomato fluorescent protein, was used as volume marker control. For experiments with recombinant TAU constructs, $\mathrm{N}$-terminally tagged $0 \mathrm{~N} 3 \mathrm{R}-\mathrm{TAU} \mathrm{HA}^{\mathrm{A}}$ and $\mathrm{N}$-term-TAUHA constructs were cloned into the same, CMV promoter-driven expression vector (see Suppl. Sequence 1). For this, the intended TAU cDNA sequences were amplified by PCR using primer pairs with restriction sites (Agel, BamHI) corresponding to the multiple cloning site of the backbone vector. Additionally, the forward primer contained a human influenza hemagglutinin (HA) tag sequence overhang with start codon (5'ATGTACCCATACGATGTTCCAGATTACGCT-3'), which was fused to the 5' site of the TAU sequence. The PCR product was analyzed via gel electrophoresis, and correctly sized inserts were cut out and purified with NucleoSpin ${ }^{\mathrm{TM}}$ Gel \& PCR Clean up Kit (Macherey \& Nagel). Vector and insert were digested (30 min, $37^{\circ} \mathrm{C}$ ) according to manufacturer's protocols (enzymes by New England Biolabs), either purified directly (insert) or from agarose gel (vector) and ligated $\left(16-18 \mathrm{~h}, 20-22^{\circ} \mathrm{C}\right.$ ) with T4 DNA ligase (NEB) according to the manufacturer's protocol.

For plasmid amplification, plasmids were transformed into chemocompetent bacteria (E. coli Top $10^{\mathrm{TM}}$, Thermo Fisher Scientific, TFS), which were generated like previously described ${ }^{70}$. Bacteria were spread onto pre-warmed agar plates with antibiotics $\left(16-18 \mathrm{~h}, 37^{\circ} \mathrm{C}\right)$, colonies were picked and grown in nutrient medium with antibiotics. For plasmid isolation and purification from bacterial suspensions, the PureYield ${ }^{\mathrm{TM}}$ MidiPrep Kit (ProMega) and NucleoBond Xtra ${ }^{\mathrm{TM}}$ Midi/Maxi Kit (Macherey \& Nagel) were used according to the manufacturer's protocols. Plasmid concentration and purity were checked spectrophotometrically (NanoDrop ${ }^{\mathrm{TM}} 100, \mathrm{TFS}$ ) and isolates were long-term stored at $-20^{\circ} \mathrm{C}$.

\section{SH-SY5Y cell cultivation and differentiation}

Maintenance. The SH-SY5Y neuroblastoma cell line was kindly provided by Prof. Dr. Rudolf Wiesner (Institute of Veg. Physiology II, University Hospital Cologne). SH-SY5Y cells were cultured in DMEM/F12 (\#10565-018, TFS) supplemented with $10 \%$ fetal bovine serum (FBS, Biochrom AG), penicillin/streptomycin and amphotericin B (1X Anti/Anti, \#15240062, TFS) (referred to as 'SHM-10') on uncoated cell culture flasks (VWR). Cultures were maintained in a sterile incubator (MCO-20AIC, PHCbi) at $37{ }^{\circ} \mathrm{C}, 95 \%$ air humidity and $5 \% \mathrm{CO}_{2}$ concentration. Culture medium was changed once or twice per week, depending on cell density, and cells were passaged for further cultivation or differentiation experiments at $70-80 \%$ confluency. For passaging, SH-SY5Y cells were washed with DPBS, trypsinized (0.05\% Trypsin/EDTA, TFS) for 3-5 minutes, spun down at $1000 \mathrm{~g}$ for 3 minutes and resuspended in SHM-10. For differentiation experiments, resuspended SH-SY5Y cells were counted with an automatic cell counter (TC20 ${ }^{\mathrm{TM}}$, Bio-Rad) and seeded with 7.5 to $10 \times 10^{3}$ cells $/ \mathrm{cm}^{2}$ onto glass coverslips (VWR), coated with $20 \mu \mathrm{g} / \mathrm{ml}$ Poly-D-lysine (PDL, AppliChem) for at least $3 \mathrm{~h}$ at $37^{\circ} \mathrm{C}$. For long-term storage, trypsinized and spun down cells were resuspended in FBS with $10 \% \mathrm{DMSO}$, cooled down with $-1{ }^{\circ} \mathrm{C} / \mathrm{min}$ in a cryo container (Mr. Frosty ${ }^{\mathrm{TM}}$, VWR) and stored at $-80^{\circ} \mathrm{C}$ or in liquid nitrogen.

Differentiation. Differentiation protocols were started $24 \mathrm{~h}$ after seeding. For RA-based differentiation, fresh SMM with $10 \mu \mathrm{M}$ retinoic acid (RA, Sigma-Aldrich) was added at day 0 (d0) and replaced every 23 days up to d7. Differentiation with RA \& the brain-derived neurotrophic factor (BDNF, Peprotech) was adapted from former protocols ${ }^{56}$. Briefly, after RA treatment up to $d 7$ as described above, cells were washed once with DPBS, and SHM-10 medium without FBS (referred to as 'SHM-0') containing $10 \mathrm{ng} / \mathrm{ml}$ BDNF was added and once replaced until d14. Differentiation protocols for Phorbol-12-myristate-13acetate (TPA)- and RA/TPA-based differentiation were adapted from former protocols ${ }^{60,71}$. In brief, fresh SHM-10 with $81 \mu \mathrm{M}$ TPA (Sigma-Aldrich) was added and replaced every 2-3 days until d9 for TPAbased differentiation. For RA \& TPA-treatment, fresh SHM-10 with $10 \mu \mathrm{M}$ RA was added, replaced after 2-3 days up to $d 4$ or $d 5$, whereupon cells were washed once with PBS and cultivated in fresh SHM-10 with $81 \mu \mathrm{M}$ TPA until $\mathrm{d} 9$ with one medium change in between. 
bioRxiv preprint doi: https://doi.org/10.1101/2020.06.26.173526; this version posted September 24, 2020. The copyright holder for this preprint (which was not certified by peer review) is the author/funder, who has granted bioRxiv a license to display the preprint in perpetuity. It is made available under aCC-BY 4.0 International license.

Two alternative long-term differentiation procedures were tested for RA, RA/TPA and TPA treatments. The first variant (variant $A$ ) included cultivation with RA (for RA \& RA/TPA protocols) and TPA (TPA protocol) in SHM-10 until d7, cultivation with RA (RA protocol) and TPA (RA/TPA \& TPA protocols) in SHM-10 with $3 \%$ FBS (SHM-3) until d9, and cultivation with RA (RA protocol) and TPA (RA/TPA \& TPA protocols) in SHM-0 until d14. The second variant (variant $B$ ), where the seeding density was increased to 15 to $20 \times 10^{3}$ cells/cm², included cultivation with RA (for RA \& RA/TPA protocols) and TPA (TPA protocol) in SHM-10 until d2, cultivation with RA (RA \& RA/TPA protocols) and TPA (TPA protocol) in SHM-3 until d7, cultivation with RA (RA protocol) and TPA (RA/TPA \& TPA protocols) in SHM-3 until d9, and cultivation with RA (RA protocol) and TPA (RA/TPA \& TPA protocols) in SHM-0 until d14. The acquired cultures showed decreased viability after transfection (see Suppl.Fig. 1).

\section{Transfection}

SH-SY5Y-derived neurons were transfected using the polymer-based PolyJet ${ }^{\mathrm{TM}}$ DNA transfection reagent (\#SL100688, SignaGen) at d5 of differentiation. Transfection was performed according to the manufacturer's protocol with the following modifications: Before transfection, conditioned medium was collected from each well and stored at $37^{\circ} \mathrm{C}$ with $5 \% \mathrm{CO}_{2}$. For one well of a 24-well plate, $0.33 \mu \mathrm{g}$ of plasmid DNA and $1 \mu \mathrm{l}$ PolyJet ${ }^{\mathrm{TM}}$ reagent were separately prepared with $25 \mu \mathrm{l}$ DMEM (\#P04-03500, TFS), then mixed, incubated and added dropwise to the cultures. Medium was changed to previously collected, conditioned medium, supplemented with fresh doses of RA or TPA, 3-16 h after transfection. Transfection efficiency ranged from 5-10\%. For experiments with recombinant TAU, tdTomato- and TAUHA construct-encoding plasmids were co-transfected in SH-SY5Y-derived neurons. For this, DNA of both plasmids was incubated commonly with PolyJet ${ }^{\mathrm{TM}}$. The plasmid DNA was mixed with a ratio of three (TAUHA plasmid) to one part (tdTomato plasmid). The total DNA amount per well was not altered, i.e. $0.33 \mu \mathrm{g}$ was used for one well of a 24-well plate.

\section{Primary neurons cultivation}

Isolation. Pregnant female FVB wild type mice were anesthetized and sacrificed at day 13.5 of pregnancy by authorized personnel with respect to the official regularities and guidelines of the governmental authority, the Landesumweltamt (LANUV) of North Rhine-Westphalia, Germany. Preparation of E13.5 embryos, and isolation and cultivation of primary neurons were performed like previously described ${ }^{72}$. In brief, embryos were decapitated, embryonic scalp, skull and meninges were removed, the cerebral hemispheres were isolated, washed, dissociated for 5-7 minutes with $0.05 \%$ Trypsin/EDTA, and homogenized in HBSS (w/o Ca ${ }^{2+} / \mathrm{Mg}^{2+}, \# 14185052$, TFS). Dissociated primary cells were counted as described above for SH-SY5Y cells and seeded with 5 to $7.5 \times 10^{4} \mathrm{cell} / \mathrm{s} / \mathrm{cm}^{2}$ in neuronal plating medium (NPM), i.e. Neurobasal ${ }^{\mathrm{TM}}$ medium (\#21103, TFS) supplemented with $1 \% \mathrm{FBS}$, penicillin/streptomycin and amphotericin B (1X Anti/Anti), GlutaMAX ${ }^{\mathrm{TM}}$ (1X, TFS) and NS-21 (1X, \#P0720001, PAN Biotech), onto glass coverslips, coated with $20 \mu \mathrm{g} / \mathrm{ml} \mathrm{PDL}$ for at least $3 \mathrm{~h}$ at $37^{\circ} \mathrm{C}$.

Maintenance. Four days after seeding, the medium was doubled by adding neuronal maintenance medium (NMM), i.e. NPM without FBS. Cytosine arabinoside (Ara-C, \#C1768, Sigma-Aldrich) was added to an end concentration of $0.5-1.0 \mu \mathrm{g} / \mathrm{ml}$ to impair survival of glial, endothelial and other proliferating cells.

Transfection. Mouse primary forebrain neurons were transfected at day 6 in vitro (div6). Transfection was performed as described previously ${ }^{73}$. In brief, $0.25 \mu \mathrm{g}$ of plasmid DNA and $0.375 \mu \mathrm{l}$ Lipofectamine ${ }^{\mathrm{TM}}$ 2000 (\#743517, Invitrogen) were mixed for one well of a 24-well plate, incubated for 30 minutes and added dropwise to the cultures. Medium was changed to previously collected, conditioned medium 60 minutes after transfection. For experiments with recombinant TAU, tdTomato- and TAUHA constructencoding plasmids were co-transfected in mouse primary neurons. For this, the DNA of both plasmids was mixed with a ratio of three parts (TAUHA DNA) to one part (tdTomato DNA) before addition of Lipofectamine $^{\text {TM }} 2000$. The total DNA amount per well was not altered, i.e. $0.25 \mu \mathrm{g}$ per 24 -well $(1 \mu \mathrm{g}$ per 6 -well) was used for one well of a 24-well plate. 
bioRxiv preprint doi: https://doi.org/10.1101/2020.06.26.173526; this version posted September 24, 2020. The copyright holder for this preprint (which was not certified by peer review) is the author/funder, who has granted bioRxiv a license to display the preprint in perpetuity. It is made available under aCC-BY 4.0 International license.

\section{Fixation \& Immunofluorescence}

Fixation and immunofluorescence of cell cultures was done as described previously ${ }^{73}$ with slight modifications. In brief, cells were fixed for $1 \mathrm{~h}$ with $3.7 \%$ formaldehyde (in PBS), permeabilized and blocked with $5 \%$ bovine serum albumin (BSA, Sigma-Aldrich) and $0.1 \%$ Triton X-100 (AppliChem) in PBS for 5 minutes, incubated with the primary antibody (diluted in PBS) either for $2-3 \mathrm{~h}$ at $20-22{ }^{\circ} \mathrm{C}$ or preferentially at $4{ }^{\circ} \mathrm{C}$ for $16-18 \mathrm{~h}$, thoroughly washed, and incubated with the corresponding secondary antibody (diluted in PBS), which was coupled to AlexaFluor ${ }^{\mathrm{TM}}$ fluorophores, for $1-2 \mathrm{~h}$ at $20-22{ }^{\circ} \mathrm{C}$. For detection of ANKG and TRIM46 levels, the fixation process was modified (30 minutes instead of $1 \mathrm{~h}$ ) according to the sensitivity of AIS proteins to fixation procedures reported in previous studies ${ }^{74,75}$. The following primary antibodies were used: rabbit anti-TAU (K9JA) (1:1000, \#A0024, DAKO), rabbit antiHA (1:1000, \#3724S, cellSignaling), mouse anti-HA (1:1000, \#901533, BioLegend), chicken anti-MAP2 (1:2000, ab5392, Abcam), mouse anti-ANKG (N106/36) (1:250, MABN466, Neuromab), rabbit antiTRIM46 (1:500, \#377003, Synaptic Systems). Nuclei were stained with NucBlue ${ }^{\mathrm{TM}}$ (1 drop/ml, Hoechst 33342, TFS) for 20-30 minutes, and samples were mounted on objective slides (\#145-0011, Bio-Rad) using aqueous PolyMount ${ }^{\mathrm{TM}}$ (\#18606, Polysciences) or Mowiol ${ }^{\mathrm{TM}}$ (AppliChem) mounting medium, dried for $24 \mathrm{~h}$ at $\mathrm{RT}$ and long-term stored at $4{ }^{\circ} \mathrm{C}$ in the dark.

\section{Microscopy}

Immunostained cells were imaged with a widefield fluorescence microscope (Axioscope 5, Zeiss), using an LED excitation lamp (Colibri 7, Zeiss), a fluorescence camera device (Axiocam 503 mono, Zeiss), objectives with $10 \times, 20 \times, 40 \times$ (air-based) and $63 \times$ (oil-based) magnification (Zeiss) and Zen imaging software (Zen Blue pro, Zeiss). Exposure time and light intensity were adjusted to avoid excessive sample bleaching and to preclude saturation of fluorescent signal detection. For the analysis of protein expression levels, all images were taken with identical settings regarding laser intensity and exposure time to ensure statistical comparability. For documentation during cell cultivation, images were taken with a brightfield light microscope (DM IL LED, Leica) using objectives with 10x and $20 \mathrm{x}$ (air-based) magnification (Leica) and Las $\mathrm{X}$ imaging software (Leica).

\section{Data analysis}

Differentiation Efficiency. The efficiency of SH-SY5Y cell differentiation was defined as relative number of SH-SY5Y-derived neurons compared to undifferentiated and non-neuronal cells. SH-SY5Y cells were considered as differentiated if they fulfilled the following morphological criteria: i) roundish shaped soma, ii) changed nuclear morphology, i.e. smaller and brighter nuclear signal, iii) axonal outgrowth exceeds soma diameter at least twofold and iv) determined axon length exceeds $50 \mu \mathrm{m}$. Further analysis was performed only with cells reaching these criteria.

Axonal outgrowth. Axonal outgrowth of SH-SY5Y-derived neurons was determined using the Fiji/lmageJ software (NIH). The processes were traced manually, and axon length was determined as pixel number, transformed to absolute distances with respect to the original image resolution. The measured section reached from the soma-to-axon-border to the most distal axonal part, which was still detectable. Included cells fulfilled the described differentiation criteria and the axon was distinctive from processes of adjacent cells.

Endogenous protein expression. For the analysis of total endogenous expression of the neuronal maturation markers TAU and MAP2, SH-SY5Y-derived neurons were analyzed with Fiji/lmageJ software. Entire cells were encircled manually, and the mean fluorescence intensity (MFIneuron) of this marked region of interest (ROI) was determined. To exclude background fluorescence, MFI of an empty area next to the neuron was determined in the same manner (MFInoise) and subtracted from the MFIneuron. Further, MFI was determined in absence of primary antibodies (MFl unspeciif), and also subtracted from MFIneuron to eliminate impact of unspecific binding of the secondary antibody. Protein levels of differentiated cells were normalized to that of undifferentiated cells. To ensure comparability of protein levels, all cell cultures of one experiment were cultivated on the same plate, immunostained and mounted in parallel with identical antibody solutions and imaged with identical microscope settings.

Axonal enrichment of endogenous TAU. By using Fiji/ImageJ, a random patch within the soma, which had no overlap with the nuclear signal, was set as first ROI for each analyzed SH-SY5Y-derived neuron. 
bioRxiv preprint doi: https://doi.org/10.1101/2020.06.26.173526; this version posted September 24, 2020. The copyright holder for this preprint (which was not certified by peer review) is the author/funder, who has granted bioRxiv a license to display the preprint in perpetuity. It is made available under aCC-BY 4.0 International license.

An axonal segment of around $5-10 \mu \mathrm{m}$ in $50-75 \mu \mathrm{m}$ distance to the soma was chosen as second ROI. TAU signals were measured as MFI for both somatic and axonal ROls (MFIsoma, MFlaxon). To exclude background fluorescence, MFI of empty areas next to the soma and axon were determined in the same manner $\left(\mathrm{MFI}_{\text {noiseSoma, }}, \mathrm{MFI}_{\text {noiseAxon }}\right.$ ) and subtracted from the $\mathrm{MFI}_{\text {soma }} / \mathrm{MFI}_{\text {axon }}$. The ratio of $\mathrm{MFI}_{\text {axon }}$ and $\mathrm{MFI}_{\text {soma }}$ was calculated (axon-to-soma-ratio). The axon-to-soma-ratio of the volume marker tdTomato was determined using identical ROIs and identical calculation steps. The axon-to-soma-ratio of TAU was normalized to this of tdTomato to achieve the axonal enrichment factor of TAU (AEFTAU). Only tdTomato-positive SH-SY5Y-derived neurons were included for analysis.

Axonal enrichment of TAU ${ }^{\mathrm{HA}}$ constructs. $\mathrm{ROI}$ selection and calculation of the axonal enrichment factor of TAUHA constructs (AEFTAU_HA) were done as described above for endogenous TAU with a few deviations. Instead of TAU signals, the intensity of anti-HA signal was measured. Only cells that were successfully co-transfected with tdTomato and TAUHA were included for analysis. Further, to overcome signal of unspecific anti-HA antibody binding, MFls of somatic and axonal ROls from untransfected cells

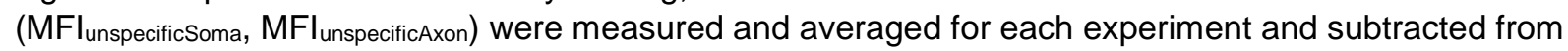
all MFIs of TAUHA from transfected cells.

Plot profiles of the proximal axon. The expression profiles of ANKG, TRIM46 and MAP2 proteins were determined at the proximal axon of SH-SY5Y-derived and mouse primary neurons. By using the $\mathrm{Fiji} /$ Image J software, the first $75 \mu \mathrm{m}$ of the axon, starting with the soma-to-axon-border, were traced with a segmented line (thickness: three pixels), and a plot profile was generated. To exclude influence of background fluorescence, a plot profile of the region right next to the axon was measured and subtracted from the original value. Further, a plot profile was determined in absence of primary antibodies and subtracted from the sample values to eliminate impact of unspecific binding of the secondary antibody.

Protein enrichment at the proximal axon. For the calculation of the protein enrichment at the proximal axon, the section of the mean plot profiles, where the fluorescent signal reached at least $50 \%$ of the maximum value, was determined in mouse primary neurons. All values within this section were averaged, separately for each individual experiment. In order to achieve the enrichment factor of ANKG andTRIM46 at the proximal axon, this average value was normalized against a baseline value of each experiment. This baseline value was acquired by averaging the most distal values of the plot profile. Here, the number of included values was equal to the value number of the previously determined section at the proximal axon. The identical sections used for mouse primary neurons were then applied to the corresponding plot profiles of SH-SY5Y-derived neurons to determine the protein enrichment of ANKG and TRIM46 in the same manner.

Statistical analysis. All statistical calculations were performed using PRISM ${ }^{\mathrm{TM}}$ analysis software (V8.3, GraphPad Inc.). For comparisons between one group of samples and a constant number (e.g. 1.0) or between two groups of samples, a two-sided student's t-test was used for the determination of $p$-values. If the standard deviation differed significantly among the two groups $(p \geq 0.05)$, the t-test was conducted with Welch's correction. When multiple groups were tested for significant differences, a Brown-Forsythetest was applied to check if the standard deviation (SD) differed significantly $(p \geq 0.05)$ among the tested groups. If this was the case, a Brown-Forsythe and Welch's ANOVA test with Dunnett's T3 correction for multiple comparisons was conducted. If the SD was not significantly different $(p<0.05)$, an ordinary one-way ANOVA test with Tukey's correction for multiple comparisons was conducted. The chosen statistical tests are mentioned for all experiments in the corresponding figure caption. The Gaussian distribution of all data sets was confirmed by a Shapiro-Wilk normality test prior to statistical analyses. Significance levels are symbolized with asterisks if $p<0.05\left(^{*}\right), p<0.01\left(^{* *}\right)$ or $p<0.001\left(^{* * *}\right), p<$ $\left.0.00011^{* * * *}\right)$. 


\section{Results}

\section{$\underline{R A / B D N F}$ treatment produces homogeneous cultures of highly polarized neurons}

We first aimed to investigate whether SH-SY5Y-derived neurons are suitable for studying TAU sorting. For this, naïve human SH-SY5Y neuroblastoma cells (Fig. 1A) were differentiated using four distinct procedures (Fig. 1B). Cells were either treated only with retinoic acid (RA) until day 7 of differentiation (d7), with RA until $\mathrm{d} 7$ followed by brain-derived neurotrophic factor (BDNF) in serum-free conditions until d14, with the phorbol ester TPA until $d 9$ or with RA until $d 4$ followed by TPA until $d 9$. To compare the amount of successfully differentiated neurons, cells were classified as differentiated when they fulfilled several criteria, e.g. roundish shaped somata and axonal processes of at least $50 \mu \mathrm{m}$ length (see methods for details). While treatment with RA (52.5 \% $\pm 11.8 \%$ neurons), TPA (39.7\% $\% 10.2 \%)$, and RA/TPA $(47.4 \% \pm$ $5.4 \%)$ generated mixed cultures of non-neuronal and neuronal cells, the sequential RA/BDNF treatment resulted in high numbers of SH-SY5Y-derived neurons $(74.1 \% \pm$ $11.4 \%$ ) (Fig. 1C\&D).

RA/BDNF-treated SH-SY5Y-derived neurons show pronounced axonal outgrowth and high levels of neuronal maturation markers

Next, the neuronal maturity of SH-SY5Y-derived neurons upon the four treatments was evaluated morphologically and biochemically. The axonal outgrowth of SH-SY5Yderived neurons was determined as the distance from the soma-to-axon-border to the distal axonal end. The average axonal outgrowth in RA/BDNF-induced neurons (264 $\mu \mathrm{m} \pm 43 \mu \mathrm{m}$ ) exceeded the axon length of all other cultures significantly, namely RA $(141 \mu \mathrm{m} \pm 21 \mu \mathrm{m}), \mathrm{RA} / \mathrm{TPA}(125 \mu \mathrm{m} \pm 28 \mu \mathrm{m})$, and TPA $(76 \mu \mathrm{m} \pm 10 \mu \mathrm{m})$, indicating the highest level of neuronal polarity in RA/BDNF-induced $\mathrm{SH}$-SY5Y-derived neurons (Fig. 2A\&C). Next, we tested the expression levels of two commonly used marker proteins for neuronal maturation ${ }^{76}$, TAU and the somatodendritic MT-associated protein 2 (MAP2) (Fig. 2B). The expression of TAU was upregulated 12-fold ( \pm 4.6 ) in RA/BDNF-treated neurons compared to undifferentiated SH-SY5Y cells. A similar increase of TAU protein levels was observed upon RA/TPA treatment (9.6-fold \pm 2.8 ). Treatment only with RA $(6.4$-fold \pm 3.3$)$ or TPA $(5.6$-fold \pm 2.6$)$ led to less strong increases of TAU levels (Fig. 2D). MAP2 levels were strongly elevated in neurons after RA/BDNF treatment (8.8-fold \pm 3.6 ), with notable inter-experimental variability, and moderately elevated after differentiation with RA (3.7-fold \pm 1.6$)$ and RA/TPA (4.1-fold $\pm 2.2)$. Treatment with TPA (1.8-fold \pm 1.1$)$ did not result in neurons with enhanced MAP2 levels (Fig. 2E). The attempt to cultivate SH-SY5Y cells in RA, TPA or RA/TPA under serum-reduced conditions were not successful, as cultures largely died upon the transfection procedure (Suppl.Fig. 1C-H), in contrast to transfected RA/BDNF-treated cultures (Suppl.Fig. 1A\&B). 
bioRxiv preprint doi: https://doi.org/10.1101/2020.06.26.173526; this version posted September 24, 2020. The copyright holder for this preprint (which was not certified by peer review) is the author/funder, who has granted bioRxiv a license to display the preprint in perpetuity. It is made available under aCC-BY 4.0 International license.

Taken together, SH-SY5Y-derived neurons, which were treated with RA/BDNF, showed the highest differentiation efficiency and pronounced neuronal maturation, indicated by axonal outgrowth and the highest expression levels of TAU and MAP2 protein.

Endogenous TAU is efficiently sorted in SH-SY5Y-derived neurons upon RA/BDNF treatment

To further evaluate the suitability of SH-SY5Y-derived neurons for TAU sorting studies, the efficiency of endogenous TAU sorting was determined. For this, the cells were transfected with tdTomato-expressing plasmids at day 5 of differentiation (d5), and the axonal enrichment factor of TAU (AEFendoTAU) was defined as axon-to-soma-ratio of TAU, normalized to the axon-to-soma-ratio of the randomly distributing volume marker tdTomato (see methods for details).

RA/BDNF-treated neurons show mainly axonal TAU and strong somatic tdTomato signals (Fig. 3A), indicating efficient axonal sorting of TAU (AEF endoTAU = $7.9 \pm 1.2)$ (Fig. 3B). For all other treatments, the SH-SY5Y-derived neurons show less axonal TAU sorting, which results in more yellowish axons in the merged images (Fig. 3A), and lower AEF endoTAU values for RA/TPA- $(3.3 \pm 0.6)$, TPA- $(3.4 \pm 0.8)$ and RA-treated neurons (Fig. 3B). The AEF endoTAU value of RA/BDNF-treated SH-SY5Y-derived neurons hints at the efficient intracellular sorting of TAU protein.

Since the results of the comparative analyses revealed RA/BDNF-treated SH-SY5Yderived neurons as the most suitable system in many respects, i.e. we observed high numbers of neurons, pronounced neuronal polarity, efficient sorting of endogenous TAU, and unchanged viability up to nine days after transfection, only those neurons were used for all further experiments.

\section{SH-SY5Y-derived neurons show robust sorting of overexpressed ON3R-TAUHA}

Efficient axonal sorting of transfected and overexpressed TAU constructs is an oftenfaced problem in primary neuron models ${ }^{50}$. Therefore, we checked whether SH-SY5Yderived neurons achieve an endogenous-like sorting efficiency of transfected human wild type TAU. For this, we co-transfected SH-SY5Y-derived neurons with tdTomato and the shortest TAU isoform ON3R, fused to an N-terminal HA tag (ON3R-TAUHA) (Fig. 4B), at d5 of differentiation. The subcellular distribution was again quantified at d14 (Fig. 4A\&C). The same experiments were done in mouse primary forebrain neurons, with co-transfection at div6 and quantification at div9 (Fig. 4D).

Strikingly, the axonal enrichment of the overexpressed ON3R-TAUHA construct (AEFTAU_HA $=7.4 \pm 1.3$ ) was similar to that of endogenous TAU in RA/BDNF-treated SH-SY5Y-derived neurons at d14 ( $94 \%$ of AEFendoTAU $=7.9 \pm 1.2)$ (Fig. 4A\&C). In mouse primary neurons, 0N3R-TAU ${ }^{H A}$ was also axonally sorted (AEFTAU_HA $=7.1 \pm$ 3.2), but less efficient than the endogenous mouse TAU ( 49 \% of AEF endoTAU $=14.5$ \pm 0.6 ) (Fig. 4D, Suppl.Fig. 2). These results demonstrate that SH-SY5Y-derived neurons are able to sort overexpressed TAU with similar efficiency as endogenous 
bioRxiv preprint doi: https://doi.org/10.1101/2020.06.26.173526; this version posted September 24, 2020. The copyright holder for this preprint (which was not certified by peer review) is the author/funder, who has granted bioRxiv a license to display the preprint in perpetuity. It is made available under aCC-BY 4.0 International license.

TAU. This makes SH-SY5Y-derived neurons suitable for addressing key questions of TAU sorting behavior, such as the role of TAU domains, modifications, or interactions within the proximal axon.

TAUHA lacking the C-terminus shows no axonal sorting in SH-SY5Y-derived and mouse primary neurons

In order to demonstrate this suitability, we next evaluated the sorting behavior of a truncated TAU ${ }^{\mathrm{HA}}$ version. As former data suggested an important role of the $\mathrm{N}$-terminus for axonal sorting ${ }^{43}$, we used a construct which lacks the entire $\mathrm{C}$-terminal half (N-termTAU ${ }^{H A}$ ) (Fig. 4B). The sorting efficiency of N-term-TAU ${ }^{H A}$ was analyzed in SH-SY5Yderived and mouse primary neurons. Interestingly, $\mathrm{N}$-term-TAU ${ }^{\mathrm{HA}}$ was axonally enriched neither in SH-SY5Y-derived neurons (AEFTAU_HA $=0.97 \pm 0.03$ ) nor in mouse primary neurons $\left(A E F_{T A U}{ }_{\text {HA }}=0.67 \pm 0.05\right)$ (Fig. 4A,C,D, Suppl.Fig. 2). Moreover, Nterm-TAU ${ }^{H A}$ tended to accumulate in the soma, indicated by HA-positive puncta in some neurons (Fig. 4A, Suppl.Fig. 2). These results, which are consistent in both used neuronal model systems, strongly suggest that the N-terminal half of TAU is either not involved in the process of axonal sorting or at least not sufficient for its successful implementation.

ANKG and TRIM46 are not enriched at the AIS region in $\mathrm{SH}-\mathrm{SY} 5 \mathrm{Y}$-derived neurons despite efficient axonal TAU sorting

Our data illustrate the pronounced neuronal polarity of SH-SY5Y-derived neurons, including the efficient axonal targeting of endogenous and transfected TAU. Since the axon initial segment (AIS) is essential for the development and maintenance of neuronal polarity ${ }^{35,37}$, we evaluated the enrichment of two AIS proteins, Ankyrin G (ANKG) and tripartite motif-containing protein 46 (TRIM46), in these cells (Fig. 5). ANKG is a master organizer of the AIS architecture as it recruits AIS-located scaffold proteins, ion channels or cytoskeletal elements ${ }^{35,37,38}$. TRIM46 is at MT-organizing protein that was shown to enrich at the proximal axon prior to ANKG and to promote the polarized distribution of TAU ${ }^{39,77}$.

In mouse primary neurons, we observed robust expression of TRIM46 and ANKG at the proximal axon, already at div9 (Fig. 5D-K) with a strong enrichment of both proteins at the proximal axon (Fig. 5M\&N). In addition to TAU (Fig. 4D, Suppl. Fig. 2), also MAP2 shows a polarized subcellular distribution, as it is tightly restricted to the somatodendritic compartment (Fig. 5L). This is in line with previous studies on neuronal polarity that suggest the ANKG-dependent AIS formation to be necessary for somatic MAP2 restriction ${ }^{35,39,78}$. The somatic retention of MAP2 is less efficient in SH-SY5Yderived neurons, indicated by the more than 3-fold increased distance of the halfmaximal intensity (SH-SY5Y: $17.7 \mu \mathrm{m}$ distal of soma-to-axon-border; primary: $5.8 \mu \mathrm{m}$ ), but not completely absent (Fig. 5I\&L). Strikingly, almost no detectable levels of ANKG were observed at the proximal axon of SH-SY5Y-derived neurons (Fig. 5A,C,G), suggesting the absence of a classical AIS. This is supported by the random-like distribution of ANKG across the axon (Fig. 5M). Further, only low TRIM46 levels were 
seen across the axon (Fig. 5B,C,H) without significant enrichment at the proximal part (Fig. 5N). This means that SH-SY5Y-derived neurons can efficiently sort endogenous TAU and overexpressed ON3R-TAU ${ }^{H A}$ without detectable enrichment of ANKG or TRIM46 at the proximal axon. Thus, the process of axonal TAU sorting in neurons may be independent of ANKG and TRIM46 accumulation at the AIS. 


\section{Discussion}

Mislocalization of the axonal TAU protein is a hallmark of Alzheimer's disease and related tauopathies. In this study, we first aimed to establish a suitable neuronal cell model, derived from SH-SY5Y cells, for studying axonal TAU sorting. We then used these SH-SY5Y-derived neurons and mouse primary forebrain neurons i) to evaluate the subcellular sorting of a C-terminus-lacking version of TAU, and ii) to test whether the efficient axonal TAU sorting in SH-SY5Y-derived neurons depends on the enrichment of ANKG and TRIM46 at the proximal axon.

\section{RA/BDNF-treated SH-SY5Y-derived neurons are suitable for TAU sorting research}

In the first step, we evaluated whether SH-SY5Y-derived neurons are a suitable model system for studying axonal TAU sorting. We found that sequential treatment with RA and BDNF generated the highest number of neurons with the most pronounced axonal outgrowth, high expression levels of TAU and MAP2 and efficient axonal sorting of TAU. As seen in former studies ${ }^{56,79,80}$, only the administration of BDNF allowed the use of serum-free media, which explains the pure and thereby stable neuronal cultures. Culture purity, neuronal polarity and TAU sorting efficiency were inferior in SH-SY5Yderived neurons treated with RA, TPA or TPA. Based on these findings, all further experiments were performed with RA/BDNF-treated SH-SY5Y-derived neurons.

An often-faced problem of rodent primary cultures is the inefficient sorting of transfected and overexpressed TAU constructs ${ }^{42,50,81}$. This effect was also observed in our study, where axonal enrichment of ON3R-TAUHA was roughly $50 \%$ less than for endogenous TAU in mouse primary neurons. In contrast, the axonal enrichment 0N3RTAU ${ }^{\text {HA }}$ reached endogenous-like levels ( $94 \%$ of endogenous TAU) in SH-SY5Yderived neurons. One explanation for this striking difference might be that SH-SY5Yderived neurons tolerate the overexpression of ON3R-TAUHA and tdTomato for at least nine days. Successfully transfected primary neurons largely died after more than three days of overexpression (data not shown). The longer expression time may facilitate the axonal targeting of ON3R-TAU ${ }^{\mathrm{HA}}$ as the plasmid DNA may become degraded after several days. This results in a reduction of protein overload and allows more efficient sorting of the exogenous protein. Another cause might be species-specific differences of the TAU sorting machinery, which affects the ability to sort human TAU isoforms in mouse primary neurons ${ }^{49}$. Further, mouse primary neurons might express a different set of isoforms that is more efficiently sorted than ON3R-TAU. However, ON3R-TAU was the most efficiently sorted isoform in former studies ${ }^{41,81}$, and ON3R-TAU is known to be the predominant isoform in early developmental stages ${ }^{3,82}$, probably including div9 neurons derived from E13.5 embryonic mice. For SH-SY5Y-derived neurons, the predominant expression of ON3R-TAU was reported ${ }^{62}$.

In brief, SH-SY5Y-derived neurons share properties of often-used neuronal models for TAU sorting as rodent primary neurons ${ }^{40-43}$ or iPSC-derived neurons ${ }^{44-48}$. But these cells overcome several of their limitations, as they are human-derived, cultivatable at low cost and without the animal need, and as a neuroblastoma cell line readily 
accessible for genetic engineering, also within the TAU-encoding MAPT gene ${ }^{68,69}$. Moreover, transfected TAU ${ }^{\mathrm{HA}}$ is sorted similar to endogenous TAU, which is difficult to achieve in rodent primary cultures ${ }^{42,50,81}$.

\section{Axonal TAU sorting requires the C-terminal half of TAU}

In the next step, we used SH-SY5Y-derived and mouse primary neurons to analyze whether TAU is still efficiently sorted when it lacks the $\mathrm{C}$-terminal half $\left(\mathrm{N}\right.$-term-TAU $\left.{ }^{H A}\right)$. No axonal enrichment of N-term-TAU ${ }^{H A}$ was seen in both used neuronal cell models. Moreover, N-term-TAU ${ }^{\mathrm{HA}}$ tends to accumulate within the soma of transfected neurons. This may hint at the increased accumulation tendency in the absence of the C-terminus or enhanced cellular degradation resulting from protein quality control mechanisms. In the latter case, N-term-TAU ${ }^{H A}$ could be enriched in subcellular compartments, such as lysosomes or stress granules. This somatic localization of N-term-TAU ${ }^{\mathrm{HA}}$ is interesting since recent studies claimed a major role for the very $\mathrm{N}$-terminal TAU domain ${ }^{43}$ and the P-rich domain ${ }^{42}$ in mediating axonal TAU sorting. As both domains are present in $\mathrm{N}$-term-TAU ${ }^{\mathrm{HA}}$, they are either not involved in TAU sorting or at least not sufficient to maintain the intracellular sorting process without the C-terminus. Notably, the Cterminal repeat domains of TAU are critical for the MT-binding affinity of the entire protein ${ }^{13,14}$. As there is evidence that MTs and MT architecture play a role in the TAU sorting process ${ }^{34,39,41}$, this could explain the necessity of the C-terminus during axonal sorting. However, recent findings question that the $\mathrm{C}$-terminal repeat domains have a strong impact on efficient TAU sorting ${ }^{42}$.

Thus, more effort is required to shed light on the TAU-intrinsic properties that ensure efficient TAU sorting. In this context, SH-SY5Y-derived neurons are valuable to conduct further and more comprehensive studies, e.g. by comparative analyses of differently truncated or modified TAU constructs.

Axonal TAU sorting is independent of ANKG and TRIM46 enrichment at the proximal axon

The formation of the AIS at the proximal part of the axon is critical for the development and maintenance of neuronal polarity, and for the polarized distribution of proteins ${ }^{35,37}$. There is strong evidence that the AIS and the integrity of its architecture play a major role in regulating the anterograde and retrograde trafficking of TAU33,34,40,41.

Therefore, we checked for the formation of an AIS in SH-SY5Y-derived neurons. We first measured the protein levels of Ankyrin G (ANKG), a master organizer of the AIS architecture $^{35-37}$, at the proximal axon of SH-SY5Y-derived and mouse primary neurons. Strikingly, no ANKG was detected within the putative AIS region of SH-SY5Yderived neurons, in contrast to the mouse primary neurons. The robust axonal sorting of endogenous and transfected TAU does apparently not require ANKG enrichment at the proximal axon or detectable ANKG expression at all although ANKG is responsible for the recruitment of major AIS structural components ${ }^{35}$. The tracing of MAP2 signal revealed invasion of MAP2 into the proximal axon. A lack of MAP2 polarity is reported for ANKG-deficient cultured neurons ${ }^{38,83}$. However, the low MAP2 levels more distal 
bioRxiv preprint doi: https://doi.org/10.1101/2020.06.26.173526; this version posted September 24, 2020. The copyright holder for this preprint (which was not certified by peer review) is the author/funder, who has granted bioRxiv a license to display the preprint in perpetuity. It is made available under aCC-BY 4.0 International license.

than $50 \mu \mathrm{m}$ clearly prove a still existing, albeit reduced, ANKG-independent somatic MAP2 retention in these cells (see Figs. 2\&5).

Recent studies revealed the tripartite motif-containing protein 46 (TRIM46), an axonal MT-organizing protein ${ }^{39,84}$, as a major player for the initial development of neuronal polarity ${ }^{39}$. TRIM46 accumulates at the proximal axon prior to ANKG and forms highly polarized MT fascicles ${ }^{38,39}$. TRIM46-deficiency leads to TAU missorting in cultured primary neurons ${ }^{77}$. Therefore, we investigated the TRIM46 levels at the proximal axon of SH-SY5Y-derived neurons. While the mouse primary neurons showed a clearly defined TRIM46 accumulation at the AIS, there were only small amounts of TRIM46 protein along the axon without a significant enrichment at the putative AIS region.

The absence of a classical AIS, indicated by the lack of ANKG and TRIM46 enrichment, together with the predominant ON3R isoform expression ${ }^{62}$ might hint at the incomplete maturity of SH-SY5Y-derived neurons ${ }^{35}$. However, SH-SY5Y-derived neurons express several neuronal maturation marker proteins ${ }^{56-62}$, and there are neuron subtypes known to lack ANKG-dependent AIS formation as well ${ }^{85}$. The spatial separation of endogenous TAU and MAP2 in SH-SY5Y-derived neurons is slightly less efficient compared to mouse primary neurons, which could be a direct consequence of the ANKG and TRIM46 absence, as both were shown to promote somatic retention of MAP2 ${ }^{83}$ and axonal sorting of TAU directly ${ }^{39,77 .}$

As ANKG and TRIM46 are absent and thus do not mediate axonal sorting of TAU in SH-SY5Y-derived neurons, the question arises, which remaining factors mediate the robust axonal targeting of TAU seen in these neurons. The end-binding protein 3 (EB3) was recently shown to be critical for the AIS integrity 86,87 . EB3 accumulates and binds along the MT network at the AIS ${ }^{87,88}$, and links it to ANKG. This EB3-ANKG interplay is necessary for the proper assembly and maintenance of the AIS by regulating the MT architecture $^{86,87}$. As EB3 also binds to the plus end of growing axons, it could directly promote anterograde trafficking of TAU and other axonal proteins via a piggyback-like mechanism ${ }^{89,90}$. Interestingly, TAU and EB3 were recently shown to co-localize at the AIS of iPSC-derived cortical neurons ${ }^{48}$. A central role in AIS assembly and maintenance was also shown for the MT-binding protein MTCL-1 ${ }^{91}$.

The relevance of TRIM46 and other MT-binding proteins in maintaining neuronal polarity ${ }^{39,86,87,91}$ strongly suggest that the organization and regulation of the MT network at the AIS is one key factor for its structural and functional integrity, which includes the correct intracellular sorting of MAP2, TAU and other compartment-specific proteins ${ }^{38}$. However, our results question the necessity of TRIM46 for the development of neuronal polarity and polarized Tau distribution. Interestingly, TAU and EB3 co-localize at the AIS of iPSC-derived cortical neurons ${ }^{48}$. It is still unclear whether this colocalization is relevant for TAU trafficking or due to the MT affinity of both proteins. Taken together, the role of MT-organizing proteins in the context of AIS integrity and axonal TAU sorting remains inconclusive and needs further elaboration, with $\mathrm{SH}$ SY5Y-derived neurons as a potential tool. 


\section{Conclusion}

In this study, we demonstrate that SH-SY5Y-derived human neurons, generated with RA/BDNF treatment, are a suitable tool for investigating TAU sorting mechanisms in human cells. These neuronal cells show pronounced neuronal polarity, neuronal marker expression, efficient sorting of endogenous and, importantly, of transfected TAU and TAU constructs similar to mouse primary neurons. By using SH-SY5Yderived neurons, we show that the $\mathrm{N}$-terminal half of TAU is not sufficient for successful axonal sorting. Further, axonal TAU targeting is independent of classical AIS formation, as ANKG and TRIM46 enrichment at the proximal axon is absent in SH-SY5Y derived neurons, but TAU is still efficiently sorted into the axon. Taken together, our data show i) that the C-terminal half of TAU is required for efficient axonal sorting, and ii) that subcellular sorting of TAU might be largely independent of classical AIS formation, which questions the claimed role of ANKG, TRIM46 and other MT-organizing proteins in developing and maintaining neuronal polarity. 


\section{Acknowledgments}

We thank Prof. Dr. Rudolf Wiesner (Institute for Veg. Physiology II, University Hospital Cologne) for providing us the SH-SY5Y neuroblastoma cell line, and Dr. Magdalena Bogus (Institute for Forensic Medicine) for cell authentication. Animals were obtained from the CMMC animal facility and the CECAD in vivo research facility (both Cologne, Germany). This work was funded by the Else-Kröner-Fresenius Stiftung, Cologne Fortune, and supported by a doctoral fellowship of the Studienstiftung des deutschen Volkes. The authors declare that they have no competing interests.

\section{Author contributions}

MB: study design, experimental conduct, data acquisition, analysis, and interpretation, manuscript drafting. SB: manuscript proofreading, assistance in methodology development. JK: experimental conduct, assistance in data acquisition and analysis. FL: assistance in experimental conduct (molecular biology). HZ: project funding, study design, data interpretation, manuscript drafting. 


\section{References}

1. Binder, L. I., Frankfurter, A. \& Rebhun, L. I. The distribution of tau in the mammalian central nervous central nervous. J. Cell Biol. (1985). doi:10.1083/jcb.101.4.1371

2. Kempf, M., Clement, A., Faissner, A., Lee, G. \& Brandt, R. Tau binds to the distal axon early in development of polarity in a microtubule- and microfilament-dependent manner. J. Neurosci. (1996).

3. Goedert, M., Spillantini, M. G., Jakes, R., Rutherford, D. \& Crowther, R. A. Multiple isoforms of human microtubule-associated protein tau: sequences and localization in neurofibrillary tangles of Alzheimer's disease. Neuron (1989). doi:10.1016/0896-6273(89)90210-9

4. Andreadis, A., Brown, W. M. \& Kosik, K. S. Structure and Novel Exons of the Human t Gene. Biochemistry (1992). doi:10.1021/bi00158a027

5. Weingarten, M. D., Lockwood, A. H., Hwo, S. Y. \& Kirschner, M. W. A protein factor essential for microtubule assembly. Proc. Natl. Acad. Sci. U. S. A. (1975). doi:10.1073/pnas.72.5.1858

6. Cleveland, D. W., Hwo, S. Y. \& Kirschner, M. W. Physical and chemical properties of purified tau factor and the role of tau in microtubule assembly. J. Mol. Biol. (1977). doi:10.1016/00222836(77)90214-5

7. Drechsel, D. N., Hyman, A. A., Cobb, M. H. \& Kirschner, M. W. Modulation of the dynamic instability of tubulin assembly by the microtubule-associated protein tau. Mol. Biol. Cell (1992). doi:10.1091/mbc.3.10.1141

8. Drubin, D. G., Feinstein, S. C., Shooter, E. M. \& Kirschner, M. W. Nerve growth factor-induced neurite outgrowth in PC12 cells involves the coordinate induction of microtubule assembly and assembly-promoting factors. J. Cell Biol. (1985). doi:10.1083/jcb.101.5.1799

9. Hirokawa, N., Shiomura, Y. \& Okabe, S. Tau proteins: the molecular structure and mode of binding on microtubules. J. Cell Biol. (1988). doi:10.1083/jcb.107.4.1449

10. Caceres, A. \& Kosik, K. S. Inhibition of neurite polarity by tau antisense oligonucleotides in primary cerebellar neurons. Nature (1990). doi:10.1038/343461a0

11. Esmaeli-Azad, B., McCarty, J. H. \& Feinstein, S. C. Sense and antisense transfection analysis of tau function: Tau influences net microtubule assembly, neurite outgrowth and neuritic stability. J. Cell Sci. (1994).

12. Dixit, R., Ross, J. L., Goldman, Y. E. \& Holzbaur, E. L. F. Differential regulation of dynein and kinesin motor proteins by tau. Science (80-. ). (2008). doi:10.1126/science.1152993

13. Butner, K. A. \& Kirschner, M. W. Tau protein binds to microtubules through a flexible array of distributed weak sites. J. Cell Biol. (1991). doi:10.1083/jcb.115.3.717

14. Goedert, M., Wischik, C. M., Crowther, R. A., Walker, J. E. \& Klug, A. Cloning and sequencing of the cDNA encoding a core protein of the paired helical filament of Alzheimer disease: Identification as the microtubule-associated protein tau. Proc. Natl. Acad. Sci. U. S. A. (1988). doi:10.1073/pnas.85.11.4051

15. Chen, J., Kanai, Y., Cowan, N. J. \& Hirokawa, N. Projection domains of MAP2 and tau determine spacings between microtubules in dendrites and axons. Nature (1992). doi:10.1038/360674a0

16. Brandt, R., Léger, J. \& Lee, G. Interaction of tau with the neural plasma membrane mediated by tau's amino-terminal projection domain. J. Cell Biol. (1995). doi:10.1083/jcb.131.5.1327

17. Gauthier-Kemper, A. et al. The frontotemporal dementia mutation R406W blocks tau's interaction with the membrane in an annexin A2-dependent manner. J. Cell Biol. (2011). doi:10.1083/jcb.201007161

18. Brion, J. P., Passareiro, H., Nunez, J. \& Flament-Durand, J. Mise en Evidence Immunologique 
de la Proteine Tau au Niveau des Lesiones de Degenerescence Neurofibrillaire de la Maladie d'Alzheimer. Arch. Biol. (Liege). (1985).

19. Wischik, C. M. et al. Isolation of a fragment of tau derived from the core of the paired helical filament of Alzheimer disease. Proc. Natl. Acad. Sci. U. S. A. (1988).

doi:10.1073/pnas.85.12.4506

20. Braak, H. \& Braak, E. Neuropathological stageing of Alzheimer-related changes. Acta Neuropathologica (1991). doi:10.1007/BF00308809

21. Selkoe, D. J. The Molecular of Alzheimer's Pathology Disease Review. Neuron (1991). doi:10.1016/0896-6273(91)90052-2

22. Arriagada, P. V., Growdon, J. H., Hedley-Whyte, E. T. \& Hyman, B. T. Neurofibrillary tangles but not senile plaques parallel duration and severity of Alzheimer's disease. Neurology (1992).

23. Braak, H., Duyckaerts, C., Braak, E. \& Piette, F. Neuropathological stageing of Alzheimer related changes correlates with psychometrically assessed intellectual status. Neurobiol. Aging (1992). doi:10.1016/0197-4580(92)90298-c

24. Ishihara, T. et al. Age-dependent emergence and progression of a tauopathy in transgenic mice overexpressing the shortest human tau isoform. Neuron (1999). doi:10.1016/S08966273(00)81127-7

25. Roy, S., Zhang, B., Lee, V. M. Y. \& Trojanowski, J. Q. Axonal transport defects: A common theme in neurodegenerative diseases. Acta Neuropathologica (2005). doi:10.1007/s00401004-0952-x

26. Zhang, B. et al. Retarded axonal transport of R406W mutant tau in transgenic mice with a neurodegenerative tauopathy. J. Neurosci. (2004). doi:10.1523/JNEUROSCI.0797-04.2004

27. Ittner, L. M. et al. Dendritic function of tau mediates amyloid- $\beta$ toxicity in alzheimer's disease mouse models. Cell (2010). doi:10.1016/j.cell.2010.06.036

28. Lacroix, B. et al. Tubulin polyglutamylation stimulates spastin-mediated microtubule severing. J. Cell Biol. (2010). doi:10.1083/jcb.201001024

29. Zempel, H., Thies, E., Mandelkow, E. \& Mandelkow, E.-M. A Oligomers Cause Localized Ca2+ Elevation, Missorting of Endogenous Tau into Dendrites, Tau Phosphorylation, and Destruction of Microtubules and Spines. J. Neurosci. (2010). doi:10.1523/jneurosci.2357-10.2010

30. Zempel, H. et al. Amyloid- $\beta$ oligomers induce synaptic damage via Tau-dependent microtubule severing by TTLL6 and spastin. EMBO J. (2013). doi:10.1038/emboj.2013.207

31. Zempel, H. \& Mandelkow, E. M. Tau missorting and spastin-induced microtubule disruption in neurodegeneration: Alzheimer Disease and Hereditary Spastic Paraplegia. Molecular Neurodegeneration 10, (2015).

32. Ittner, A. \& Ittner, L. M. Dendritic Tau in Alzheimer's Disease. Neuron (2018). doi:10.1016/j.neuron.2018.06.003

33. Zempel, H. \& Mandelkow, E. Mechanisms of Axonal Sorting of Tau and Influence of the Axon Initial Segment on Tau Cell Polarity. in Advances in Experimental Medicine and Biology (2019). doi:10.1007/978-981-32-9358-8_6

34. Scholz, T. \& Mandelkow, E. Transport and diffusion of Tau protein in neurons. Cellular and Molecular Life Sciences (2014). doi:10.1007/s00018-014-1610-7

35. Rasband, M. N. The axon initial segment and the maintenance of neuronal polarity. Nature Reviews Neuroscience (2010). doi:10.1038/nrn2852

36. Jones, S. L. \& Svitkina, T. M. Axon Initial Segment Cytoskeleton: Architecture, Development, and Role in Neuron Polarity. Neural Plasticity (2016). doi:10.1155/2016/6808293

37. Leterrier, C. The Axon Initial Segment, 50 Years Later: A Nexus for Neuronal Organization and Function. Curr. Top. Membr. (2016). doi:10.1016/bs.ctm.2015.10.005 
38. Leterrier, C. The axon initial segment: An updated viewpoint. J. Neurosci. (2018). doi:10.1523/JNEUROSCI.1922-17.2018

39. Van Beuningen, S. F. B. et al. TRIM46 Controls Neuronal Polarity and Axon Specification by Driving the Formation of Parallel Microtubule Arrays. Neuron (2015). doi:10.1016/j.neuron.2015.11.012

40. Li, X. et al. Novel diffusion barrier for axonal retention of Tau in neurons and its failure in neurodegeneration. EMBO J. (2011). doi:10.1038/emboj.2011.376

41. Zempel, $\mathrm{H}$. et al. Axodendritic sorting and pathological missorting of Tau are isoform-specific and determined by axon initial segment architecture. J. Biol. Chem. (2017). doi:10.1074/jbc.M117.784702

42. Iwata, M., Watanabe, S., Yamane, A., Miyasaka, T. \& Misonou, H. Regulatory mechanisms for the axonal localization of tau protein in neurons. Mol. Biol. Cell (2019). doi:10.1091/mbc.E1903-0183

43. Gauthier-Kemper, A. et al. Annexins $\mathrm{A} 2$ and $\mathrm{A} 6$ interact with the extreme $\mathrm{N}$ terminus of tau and thereby contribute to tau's axonal localization. J. Biol. Chem. (2018). doi:10.1074/jbc.RA117.000490

44. Muratore, C. R. et al. The familial alzheimer's disease APPV717I mutation alters APP processing and Tau expression in iPSC-derived neurons. Hum. Mol. Genet. (2014). doi:10.1093/hmg/ddu064

45. Verheyen, A. et al. Using human iPSC-derived neurons to model TAU aggregation. PLoS One (2015). doi:10.1371/journal.pone.0146127

46. Silva, M. C. et al. Human iPSC-Derived Neuronal Model of Tau-A152T Frontotemporal Dementia Reveals Tau-Mediated Mechanisms of Neuronal Vulnerability. Stem Cell Reports (2016). doi:10.1016/j.stemcr.2016.08.001

47. Wang, C. et al. Scalable Production of iPSC-Derived Human Neurons to Identify Tau-Lowering Compounds by High-Content Screening. Stem Cell Reports (2017). doi:10.1016/j.stemcr.2017.08.019

48. Sohn, P. D. et al. Pathogenic Tau Impairs Axon Initial Segment Plasticity and Excitability Homeostasis. Neuron (2019). doi:10.1016/j.neuron.2019.08.008

49. Janke, C. et al. Phylogenetic diversity of the expression of the microtubule-associated protein tau: Implications for neurodegenerative disorders. Mol. Brain Res. (1999). doi:10.1016/S0169328X(99)00079-0

50. Xia, D., Gutmann, J. M. \& Gotz, J. Mobility and subcellular localization of endogenous, geneedited Tau differs from that of over-expressed human wild-type and P301L mutant Tau. Sci. Rep. (2016). doi:10.1038/srep29074

51. Hu, B. Y. et al. Neural differentiation of human induced pluripotent stem cells follows developmental principles but with variable potency. Proc. Natl. Acad. Sci. U. S. A. (2010). doi:10.1073/pnas.0910012107

52. Gunhanlar, N. et al. A simplified protocol for differentiation of electrophysiologically mature neuronal networks from human induced pluripotent stem cells. Mol. Psychiatry (2017). doi:10.1038/mp.2017.56

53. Bell, S. et al. Differentiation of Human Induced Pluripotent Stem Cells (iPSCs) into an Effective Model of Forebrain Neural Progenitor Cells and Mature Neurons. BIO-PROTOCOL (2019). doi:10.21769/bioprotoc.3188

54. Biedler, J. L. \& Schachner, M. Multiple Neurotransmitter Synthesis by Human Neuroblastoma Cell Lines and Clones. Cancer Res. (1978).

55. Kovalevich, J. \& Langford, D. Considerations for the use of SH-SY5Y neuroblastoma cells in neurobiology. Methods Mol. Biol. (2013). doi:10.1007/978-1-62703-640-5_2

56. Encinas, M. et al. Sequential treatment of SH-SY5Y cells with retinoic acid and brain-derived 
neurotrophic factor gives rise to fully differentiated, neurotrophic factor-dependent, human neuron-like cells. J. Neurochem. (2000). doi:10.1046/j.1471-4159.2000.0750991.x

57. Cheung, Y. T. et al. Effects of all-trans-retinoic acid on human SH-SY5Y neuroblastoma as in vitro model in neurotoxicity research. Neurotoxicology (2009). doi:10.1016/j.neuro.2008.11.001

58. Lopes, F. M. et al. Comparison between proliferative and neuron-like SH-SY5Y cells as an in vitro model for Parkinson disease studies. Brain Res. (2010). doi:10.1016/j.brainres.2010.03.102

59. Xie, H. R., Hu, L. Sen \& Li, G. Y. SH-SY5Y human neuroblastoma cell line: In vitro cell model of dopaminergic neurons in Parkinson's disease. Chinese Medical Journal (2010). doi:10.3760/cma.j.issn.0366-6999.2010.08.021

60. Påhlman, S., Ruusala, A. I., Abrahamsson, L., Mattsson, M. E. K. \& Esscher, T. Retinoic acidinduced differentiation of cultured human neuroblastoma cells: a comparison with phorbolesterinduced differentiation. Cell Differ. (1984). doi:10.1016/0045-6039(84)90038-1

61. Gimenez-Cassina, A., Lim, F. \& Diaz-Nido, J. Differentiation of a human neuroblastoma into neuron-like cells increases their susceptibility to transduction by herpesviral vectors. J. Neurosci. Res. (2006). doi:10.1002/jnr.20976

62. Agholme, L., Lindström, T., Kgedal, K., Marcusson, J. \& Hallbeck, M. An in vitro model for neuroscience: Differentiation of SH-SY5Y cells into cells with morphological and biochemical characteristics of mature neurons. J. Alzheimer's Dis. (2010). doi:10.3233/JAD-2010-091363

63. Lee, M., McGeer, E. \& McGeer, P. L. Activated human microglia stimulate neuroblastoma cells to upregulate production of beta amyloid protein and tau: Implications for Alzheimer's disease pathogenesis. Neurobiol. Aging (2015). doi:10.1016/j.neurobiolaging.2014.07.024

64. Smith, C. J., Anderton, B. H., Davis, D. R. \& Gallo, J. M. Tau isoform expression and phosphorylation state during differentiation of cultured neuronal cells. FEBS Lett. (1995). doi:10.1016/0014-5793(95)01221-Y

65. Tanaka, T., Iqbal, K., Trenkner, E., Dong Jie Liu \& Grundke-lqbal, I. Abnormally phosphorylated tau in SY5Y human neuroblastoma cells. FEBS Lett. (1995). doi:10.1016/00145793(95)00061-D

66. Majd, S., Koblar, S. \& Power, J. Compound C enhances tau phosphorylation at Serine396 via PI3K activation in an AMPK and rapamycin independent way in differentiated SH-SY5Y cells. Neurosci. Lett. (2018). doi:10.1016/j.neulet.2018.01.049

67. Majd, S., Majd, Z., Koblar, S. \& Power, J. Beta estradiol and norepinephrine treatment of differentiated SH-SY5Y cells enhances tau phosphorylation at (Ser396) and (Ser262) via AMPK but not mTOR signaling pathway. Mol. Cell. Neurosci. (2018). doi:10.1016/j.mcn.2018.02.004

68. Bell, M. \& Zempel, H. SH-SY5Y-derived Neurons: A Neuronal Model System for Investigating TAU Sorting Mechanisms and Neuronal Subtype-specific TAU Vulnerability. Preprints.org (2020). doi:10.20944/PREPRINTS202006.0203.V1

69. Sola, M. et al. Tau affects P53 function and cell fate during the DNA damage response. Nat. Commun. Biol. 3, 245 (2020).

70. Sambrook, J. \& Russell, D. W. The Inoue Method for Preparation and Transformation of Competent E. Coli : "Ultra-Competent" Cells. Cold Spring Harb. Protoc. (2006). doi:10.1101/pdb.prot3944

71. Påhlman, S., Odelstad, L., Larsson, E., Grotte, G. \& Nilsson, K. Phenotypic changes of human neuroblastoma cells in culture induced by 12-O-tetradecanoyl-phorbol-13-acetate. Int. J. Cancer (1981). doi:10.1002/ijc.2910280509

72. Zempel, H. \& Mandelkow, E. M. Tracking Tau in neurons: How to grow, fix, and stain primary neurons for the investigation of Tau in all developmental stages. in Methods in Molecular Biology (2017). doi:10.1007/978-1-4939-6598-4_20 
73. Zempel, H., Luedtke, J. \& Mandelkow, E. M. Tracking Tau in neurons: How to transfect and track exogenous tau into primary neurons. in Methods in Molecular Biology (2017). doi:10.1007/978-1-4939-6598-4_21

74. Sohn, P. D. et al. Acetylated tau destabilizes the cytoskeleton in the axon initial segment and is mislocalized to the somatodendritic compartment. Mol. Neurodegener. 11, (2016).

75. Di Re, J., Kayasandik, C., Botello-Lins, G., Labate, D. \& Laezza, F. Imaging of the Axon Initial Segment. Curr. Protoc. Neurosci. (2019). doi:10.1002/cpns.78

76. Tanapat, P. Neuronal Cell Markers. Mater. Methods (2013). doi:10.13070/mm.en.3.196

77. Kneynsberg, A., Vega, I. \& Kanaan, N. M. TRIM46 Knockdown Causes Neuronal Tau Redistribution and Increases Axosomatic Tau Diffusion. Alzheimer's Dement. (2019). doi:10.1016/j.jalz.2019.06.4852

78. Barnes, A. P. \& Polleux, F. Establishment of Axon-Dendrite Polarity in Developing Neurons. Annu. Rev. Neurosci. (2009). doi:10.1146/annurev.neuro.31.060407.125536

79. Arcangeli, A. et al. Modulation of HERG current and herg gene expression during retinoic acid treatment of human neuroblastoma cells: Potentiating effects of BDNF. J. Neurobiol. (1999). doi:10.1002/(SICI)1097-4695(199908)40:2<214::AID-NEU7>3.0.CO;2-0

80. Shipley, M. M., Mangold, C. A. \& Szpara, M. L. Differentiation of the SH-SY5Y human neuroblastoma cell line. J. Vis. Exp. (2016). doi:10.3791/53193

81. Bachmann, S., Bell, M., Klimek, J. \& Zempel, H. Subcellular localization of TAU isoforms and their influence on microtubule dynamics. bioRxiv 2020.06.16.154757 (2020). doi:10.1101/2020.06.16.154757

82. Trabzuni, D. et al. MAPT expression and splicing is differentially regulated by brain region: Relation to genotype and implication for tauopathies. Hum. Mol. Genet. (2012). doi:10.1093/hmg/dds238

83. Hedstrom, K. L., Ogawa, Y. \& Rasband, M. N. AnkyrinG is required for maintenance of the axon initial segment and neuronal polarity. J. Cell Biol. (2008). doi:10.1083/jcb.200806112

84. Short, K. M. \& Cox, T. C. Subclassification of the RBCC/TRIM superfamily reveals a novel motif necessary for microtubule binding. J. Biol. Chem. (2006). doi:10.1074/jbc.M512755200

85. Gumy, L. F. et al. MAP2 Defines a Pre-axonal Filtering Zone to Regulate KIF1- versus KIF5Dependent Cargo Transport in Sensory Neurons. Neuron (2017). doi:10.1016/j.neuron.2017.03.046

86. Fréal, A. et al. Cooperative Interactions between $480 \mathrm{kDa}$ Ankyrin-G and EB proteins assemble the axon initial segment. J. Neurosci. (2016). doi:10.1523/JNEUROSCI.3219-15.2016

87. Leterrier, $\mathrm{C}$. et al. End-binding proteins EB3 and EB1 link microtubules to ankyrin $\mathrm{G}$ in the axon initial segment. Proc. Natl. Acad. Sci. U. S. A. (2011). doi:10.1073/pnas.1018671108

88. Nakata, T. \& Hirokawa, N. Microtubules provide directional cues for polarized axonal transport through interaction with kinesin motor head. J. Cell Biol. (2003). doi:10.1083/jcb.200302175

89. Stepanova, T. et al. Visualization of microtubule growth in cultured neurons via the use of EB3GFP (end-binding protein 3-green fluorescent protein). J. Neurosci. 23, 2655-2664 (2003).

90. Akhmanova, A. \& Steinmetz, M. O. Control of microtubule organization and dynamics: Two ends in the limelight. Nature Reviews Molecular Cell Biology (2015). doi:10.1038/nrm4084

91. Satake, T. et al. MTCL1 plays an essential role in maintaining Purkinje neuron axon initial segment. EMBO J. (2017). doi:10.15252/embj.201695630 


\section{Figures}

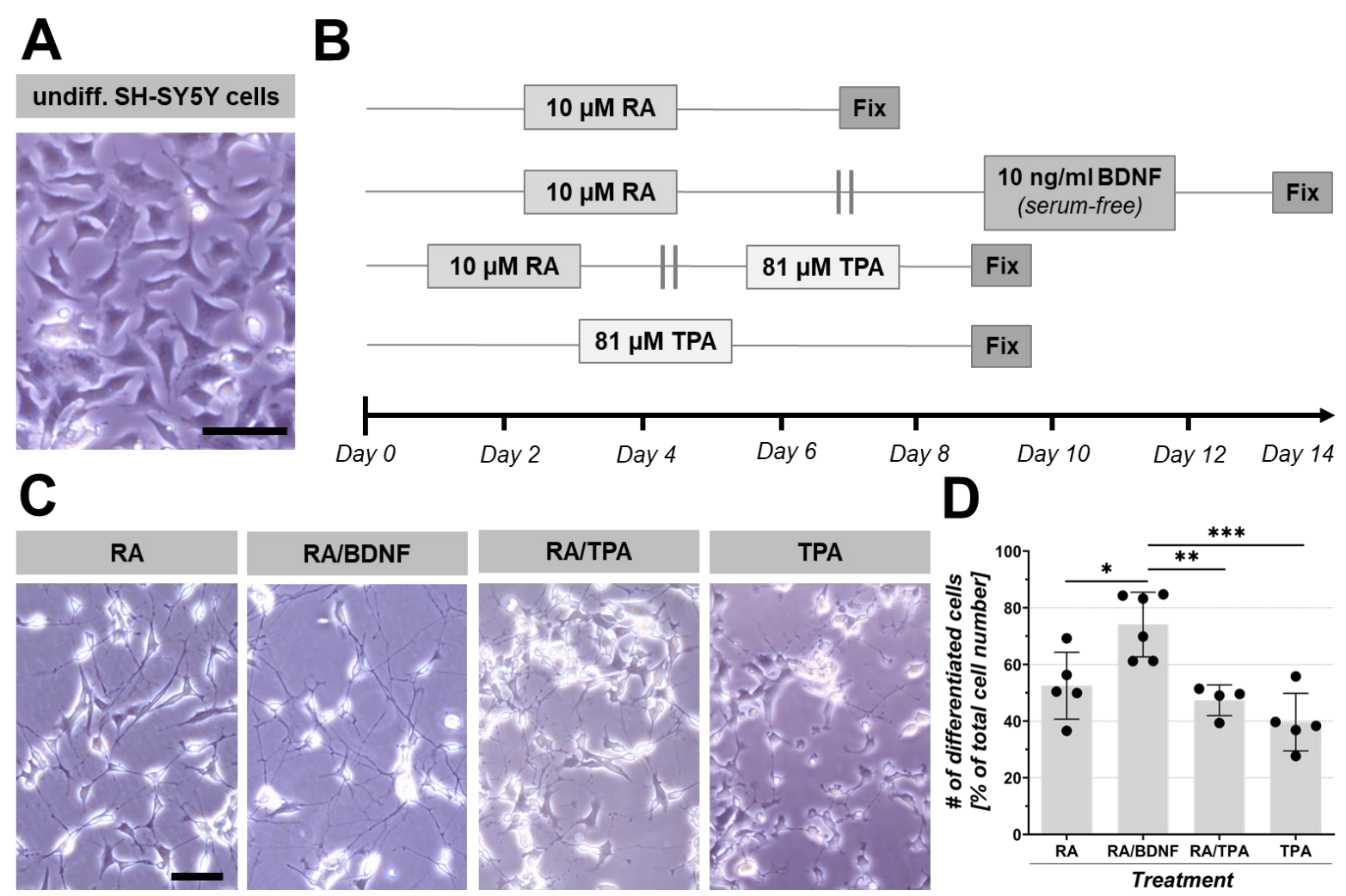

Figure 1: Generation of SH-SY5Y-derived neurons. Representative images of undifferentiated SHSY5Y cells, timeline of differentiation procedures (see methods for details), and differentiated SH-SY5Yderived neurons. A: Undifferentiated SH-SY5Y cells cultured in normal SHM-10 medium. Scale bar: 50 $\mu \mathrm{m}$. B: Schematic time course of differentiation protocols. The bottom line indicates the day (d) of differentiation. B.1: RA was applied until d7 in SH-SY5Y cell culture medium with $10 \%$ FBS (SHM-10). B.2: Sequential treatment with RA in SHM-10 until d7 and BDNF in serum-free SHM-0 medium until d14. B.3: Sequential treatment of RA in SHM-10 until d4 and TPA in SHM-10 until d9. B.4: Application of TPA in SHM-10 until d9. Grey doubled lines indicate substance changes, grey box represents fixation of cells. C: SH-SY5Y-derived neurons after differentiation with RA, RA/BDNF, RA/TPA or TPA in culture. Note i) the neurite outgrowth of SH-SY5Y-derived neurons upon all treatments and ii) the different extent of co-cultured undifferentiated cells. Scale bar: $50 \mu \mathrm{m}$. E: Quantification of differentiation efficiency. Quantification was done for four to six independent experiments with $1000 \pm 50$ cells per experiment. Differentiated cells were determined with the differentiation criteria (see methods for details). Black dots represent independent experiments, grey bars indicate the arithmetic mean of all experiments, and error bars show SD. An ordinary one-way ANOVA with Tukey's correction for multiple comparisons was performed to determine significance levels between all four methods. Significance levels: ${ }^{*} p<0.05$, ${ }^{*}$ $p<0.01,{ }^{* *} p<0.001$. 


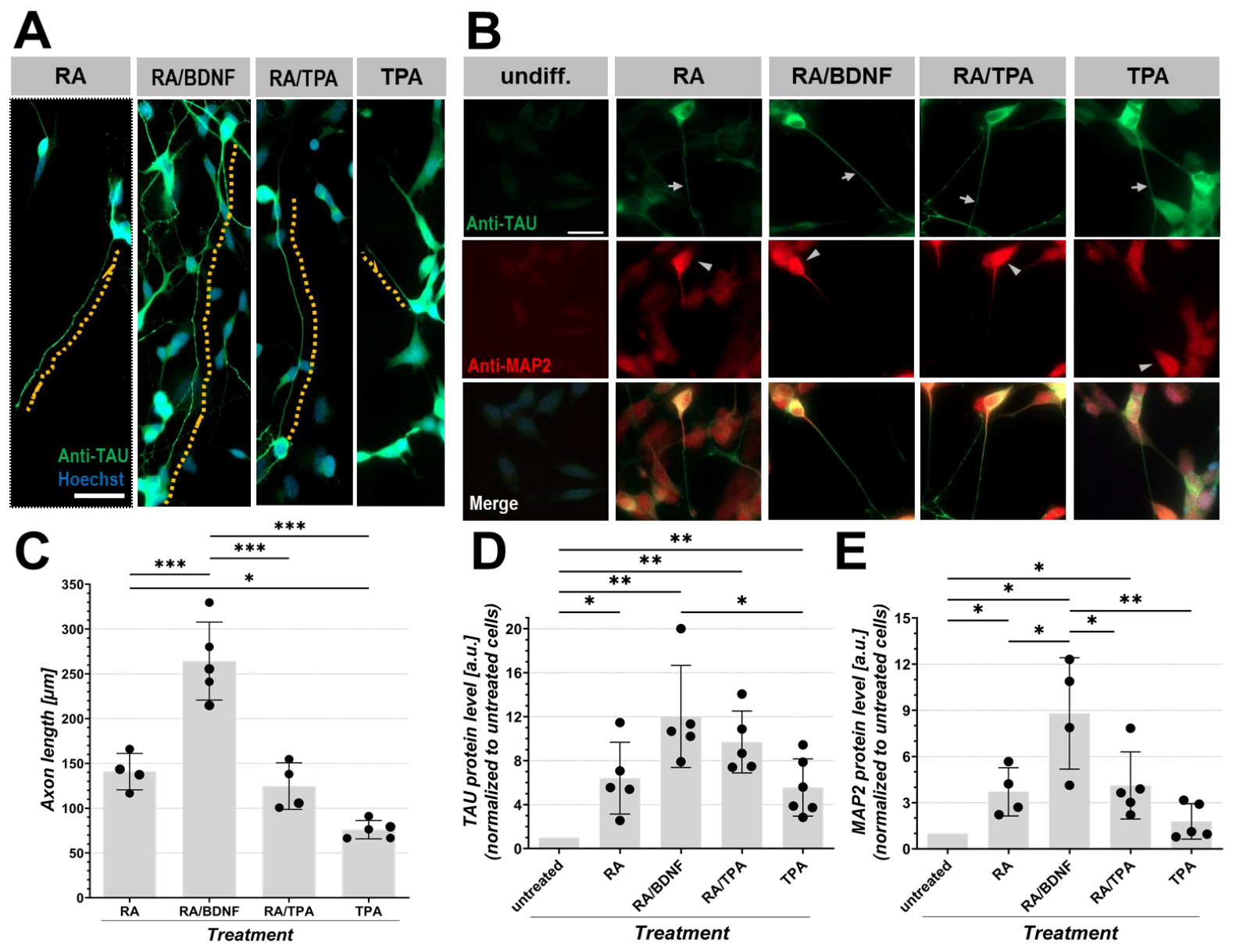

Fig. 2: Neuronal maturation of SH-SY5Y-derived neurons upon differentiation. A: Axonal outgrowth of SH-SY5Y-derived neurons after differentiation with RA, RA/BDNF, RA/TPA or TPA, immunostained with polyclonal anti-TAU (K9JA) antibody and NucBlue ${ }^{\mathrm{TM}}$ (nuclear staining, Hoechst 33342). Dashed lines trace representative axons. Note the different extent of axonal elongation. Scale bar: $50 \mu \mathrm{m}$. B: Representative images of undifferentiated SH-SY5Y and SH-SY5Y-derived neurons immunostained with polyclonal anti-TAU (K9JA) and polyclonal anti-MAP2 antibodies. TAU (green) and MAP2 (red) are shown in single channels and merged. Note the weak signal of neuronal maturation markers TAU (green) and MAP2 (red) in undifferentiated cells (left panel) and the enhanced signals after differentiation as indicated. Scale bar: $20 \mu \mathrm{m}$; C-E: Analysis of neuronal maturation in SH-SY5Yderived neurons, which were determined by nuclear and somatic shape, and neuritic outgrowth (see differentiation criteria (methods) for details). Quantification of axonal outgrowth (C) was done for four to five independent experiments with 30-35 cells traced per experiment. Quantification for TAU (D) and MAP2 (E) protein levels was done for five to six (D) or four to five $(E)$ independent experiments with 2530 cells per experiment. Black dots represent independent experiments, grey bars indicate the arithmetic mean of all experiments, and error bars show SD. An ordinary one-way ANOVA with Tukey's correction for multiple comparisons was performed to determine significance levels between all four treatments $(C)$, and all four treatments and undifferentiated cells (D\&E). Significance levels: ${ }^{*} p<0.05,{ }^{* *} p<0.01$, *** $p<0.001$. 
A


E
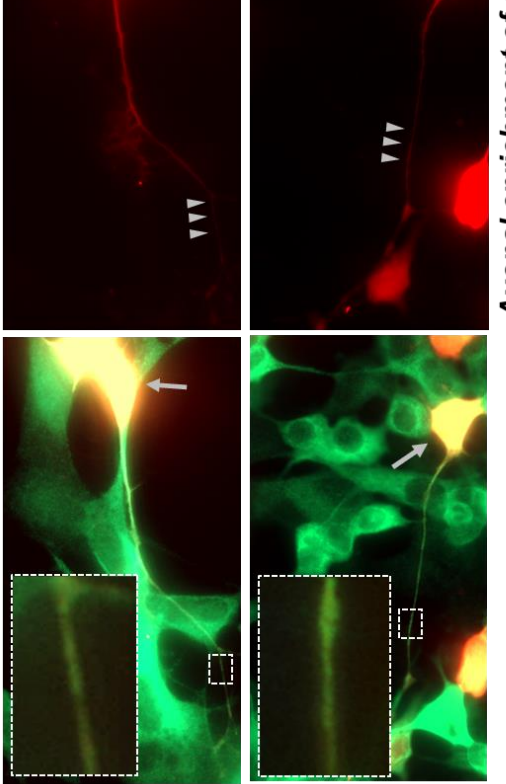

Figure 3: Axonal enrichment of endogenous TAU in SH-SY5Y-derived neurons. SH-SY5Y-derived neurons were transfected with tdTomato and immunostained with a polyclonal anti-TAU (K9JA) antibody. A: Endogenous TAU (green) and transfected tdTomato as volume marker (red) are shown in single channels and merged. Note the strong axonal sorting of TAU compared to tdTomato in SH-SY5Yderived neurons after RA/BDNF treatment (arrowheads), which leads to yellowish somatic staining (arrows) and mainly green axonal TAU staining in merged images. Axonal sections (small dashed boxes) are shown with higher magnification (large dashed boxes) in merged images. Scale bar: $20 \mu \mathrm{m}$, scale bar within dashed box: $3 \mu \mathrm{m}$. B: Quantification of the axonal enrichment of endogenous TAU

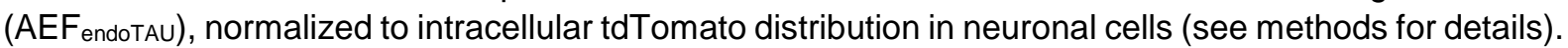
Quantification was done for three to five independent experiments with 20-30 cells per experiment. Black dots represent independent experiments, grey bars indicate the arithmetic mean of all experiments, and error bars show SD. An ordinary one-way ANOVA with Tukey's correction for multiple comparisons was performed to determine significance levels between all conditions. Significance level: ${ }^{* \star *} p<0.001$. 

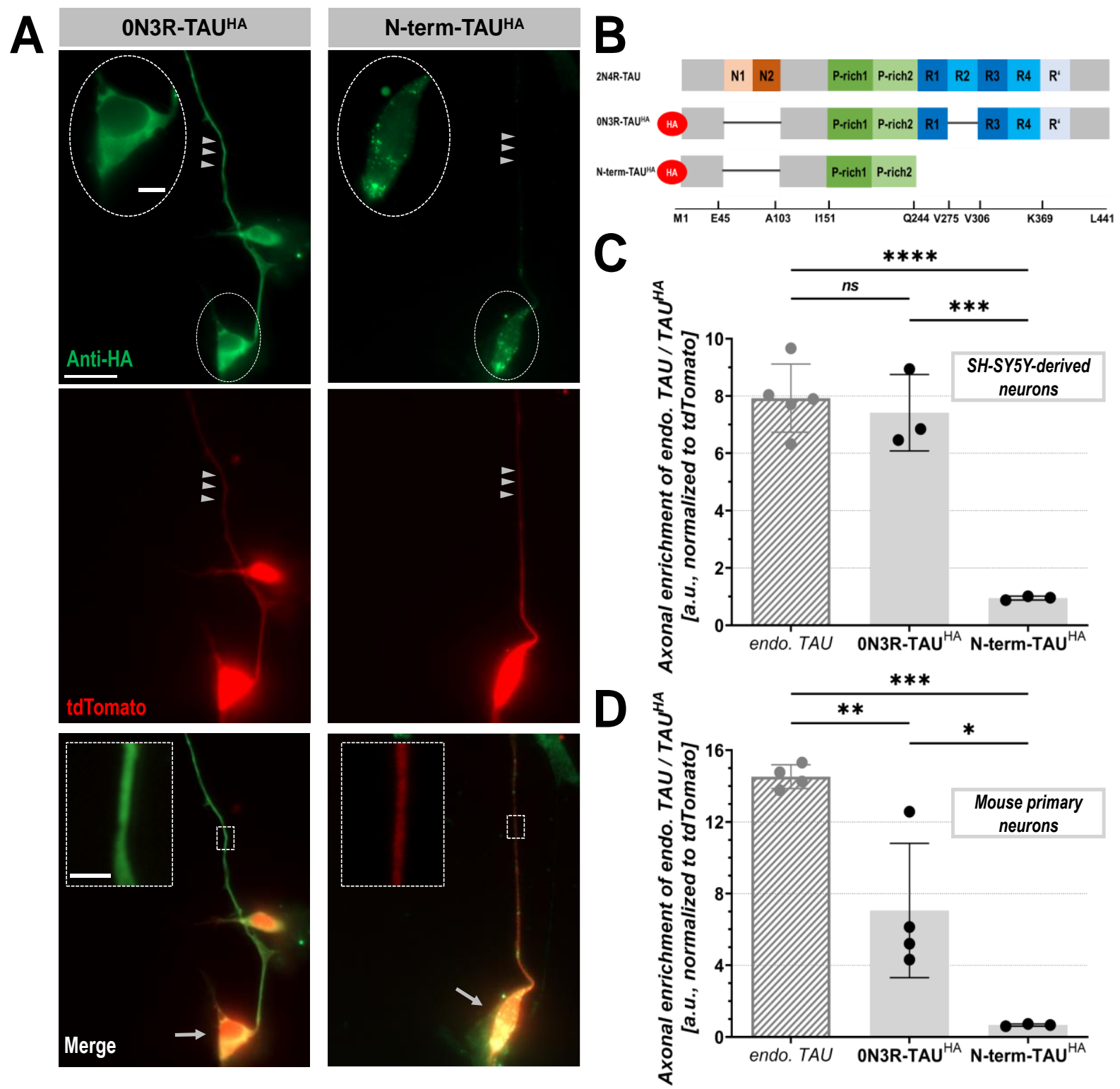

Figure 4: Axonal enrichment of transfected TAU ${ }^{H A}$ constructs in RA/BDNF-treated SH-SY5Yderived and mouse primary neurons. A: Representative immunofluorescent images of SH-SY5Yderived neurons transfected with tdTomato and either ON3R-TAUHA (left column) or N-term-TAUHA (right column). TAUHA constructs (green) and co-transfected tdTomato as volume marker (red) are shown in single channels and merged. Cells were transfected at $\mathrm{d} 5$ and fixed at $\mathrm{d} 14$ (see methods for details). Note the strong axonal sorting of TAU compared to tdTomato (arrowheads) in ON3R-TAUHA which leads to yellowish somatic staining (arrows) and mainly green axonal staining in merged images. Axons of cells with $\mathrm{N}$-term-TAUHA appear red, indicating weak axonal sorting of $\mathrm{N}$-term-TAUHA. Axonal sections (small dashed boxes) are shown with higher magnification (large dashed boxes). HA-positive puncta (small dashed circle, magnified in large dashed circle) appear in N-term-TAUHA transfected cells but not in cells expressing ON3R-TAUHA . Scale bar: $20 \mu \mathrm{m}$, scale bar within dashed circle: $5 \mu \mathrm{m}$, scale bar within dashed box: $3 \mu \mathrm{m}$. B: Domain structure of full-length 2N4R-TAU compared to the used TAU constructs. ON3R-TAUHA contains the amino acids M1 - E45, A103 - V275 and V306 - L441. N-term-TAUHA contains the amino acids M1 - E45 and A103 - V244. Missing parts are shown as black lines. Both constructs were $\mathrm{N}$-terminally fused to an HA tag (red circle). The bottom line indicates selected amino acid positions. N1/N2: N-terminal inserts, P-rich domain: Proline-rich domain, R1 - R4: C-terminal repeat domains. R': Pseudo-repeat domain. C\&D: Quantification of axonal enrichment of TAUHA constructs (AEFTAU_HA) in SH-SY5Y-derived neurons $(C)$ and mouse primary neurons (D, see Fig. S2 for corresponding images), normalized to intracellular tdTomato distribution. Quantification was done for 
bioRxiv preprint doi: https://doi.org/10.1101/2020.06.26.173526: this version posted September 24, 2020. The copyright holder for this preprint (which was not certified by peer review) is the author/funder, who has granted bioRxiv a license to display the preprint in perpetuity. It is made available under aCC-BY 4.0 International license.

three independent experiments with 20-55 cells per experiment (C), or for three to four independent experiments with 16-46 cells per experiment (D). The quantification of AEF endoTAU in SH-SY5Y-derived neurons is included for comparison (striped bar, see Fig. 3A). Black dots represent independent experiments, grey bars indicate the arithmetic mean of all experiments, and error bars show SD. An ordinary one-way ANOVA with Tukey's correction for multiple comparisons was performed to determine significance levels between endogenous TAU and the TAUHA constructs. Significance levels: ${ }^{*} p<0.05$, ${ }^{* *} p<0.01,{ }^{* * *} p<0.001,{ }^{* * *} p<0.0001, n s: p \geq 0.05$. 

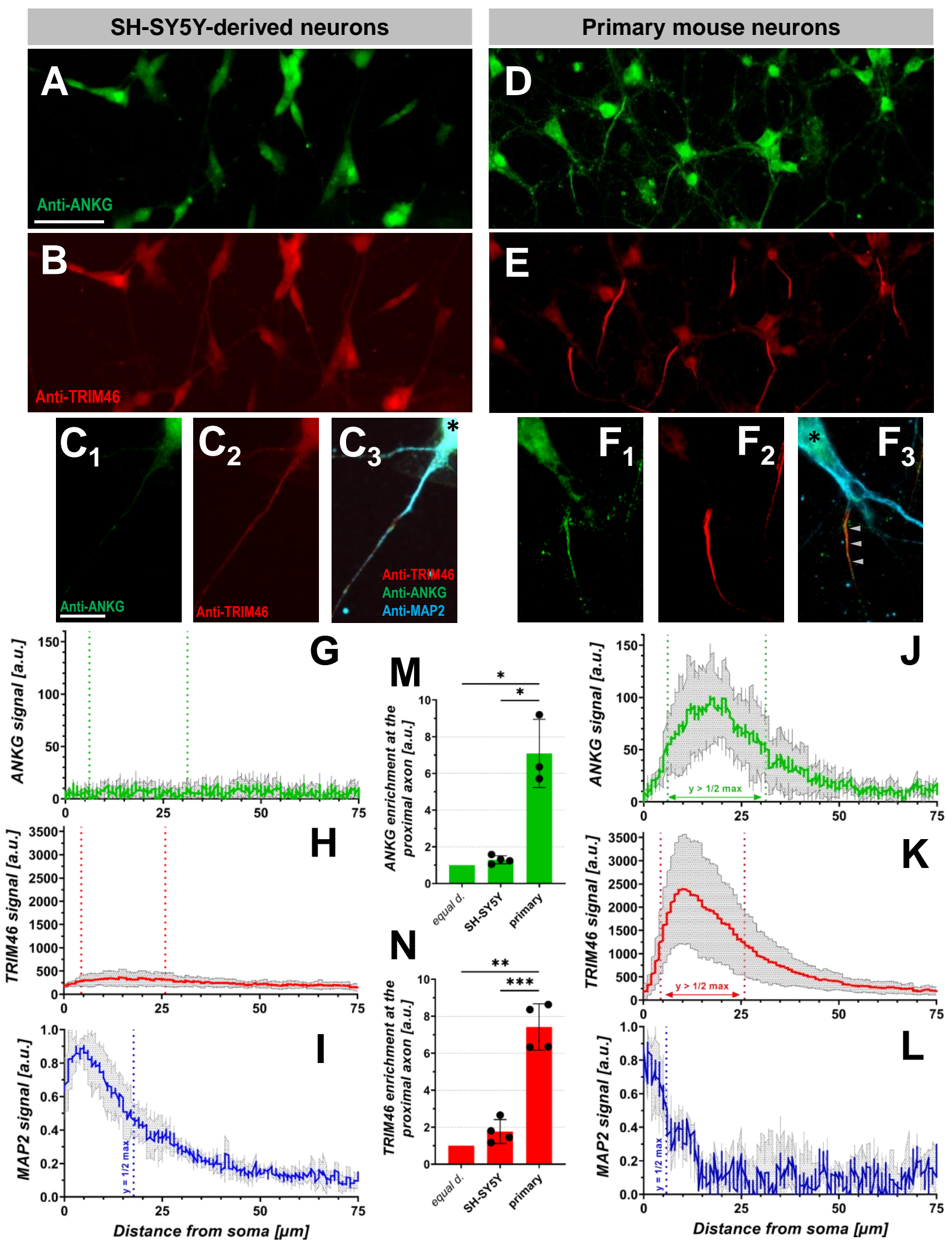

Figure 5: Formation of the AIS in SH-SY5Y-derived and mouse primary neurons. A-F: Representative images of ANKG and TRIM46 expression in SH-SY5Y-derived neurons (A-C) and mouse primary neurons (D-F), immunostained with monoclonal anti-ANKG (N106/36), polyclonal rabbit anti-TRIM46, and polyclonal anti-MAP2 antibodies. A,B\&D,E: Overview of immunostained neuronal cultures. Scale bar: $50 \mu \mathrm{m}$. C\&F: Magnification of one immunostained SH-SY5Y-derived (C) and mouse primary neuron $(F)$. Note the clearly defined enrichment of TRIM46 and ANKG at the proximal axon of mouse primary neurons (arrowheads, $F_{3}$ ), which is not visible at the proximal axon (axon defined by decreasing MAP2 intensity and process outgrowth $>75 \mu \mathrm{m}$ ) of SH-SY5Y-derived neurons. Asterisks indicate nucleus position. Scale bar (A): $20 \mu \mathrm{m}$, scale bar (C.1): $5 \mu \mathrm{m}$. G-L: Plot profiles of TRIM46 
bioRxiv preprint doi: https://doi.org/10.1101/2020.06.26.173526; this version posted September 24, 2020. The copyright holder for this preprint (which was not certified by peer review) is the author/funder, who has granted bioRxiv a license to display the preprint in perpetuity. It is made available under aCC-BY 4.0 International license.

(G\&J), ANKG (H\&K), and MAP2 (I\&L) signals at the proximal axon of SH-SY5Y-derived (G-I) and mouse primary neurons $(\mathrm{J}-\mathrm{L})$. The colored lines indicate the arithmetic mean of three to four independent experiments with 28-36 traced cells per experiment (see methods for details). The grey area shows SD. G,H\&J,K: The green and red dotted lines mark the corridor where the ANKG/TRIM46 signal are above $50 \%$ of the maximum value (determined for the signals of mouse primary neurons in J\&K). I\&L: The MAP2 plot profiles were normalized to 1 prior to analysis. The blue dotted lines indicate the distance where the MAP2 signal reaches $50 \%$ of the maximum value. M\&N: Enrichment of ANKG (M) and TRIM46 $(\mathrm{N})$ at the proximal axon (i.e. within the marked corridor), normalized to the baseline (see methods for details). Equal $d$. = equal distribution (reference value $=1$ ). Quantification was done for three to four independent experiments with 28-36 cells per experiment. Black dots represent independent experiments, colored bars indicate the arithmetic mean of all experiments, and error bars show SD. A two-sided t-test was performed to determine significance levels between the cell models and 1. A two-sided t-test with Welch's correction was performed to determine significance levels between both cell models. Significance levels: ${ }^{*} p<0.05,{ }^{* *} p<0.01,{ }^{* * *} p<0.001$. 